particularly amongst FAM participants. Preliminary effectiveness indicates potential changes in adults, but not in children, warranting further investigation.

\section{P70 BODY SIZE AND COMPOSITION, PHYSICAL ACTIVITY AND SEDENTARY TIME IN RELATION TO ENDOGENOUS HORMONES IN PRE- AND POST-MENOPAUSAL WOMEN: FINDINGS FROM THE UK BIOBANK}

S Tin Tin*, GK Reeves, TJ Key. Cancer Epidemiology Unit, Nuffield Department of Population Health, The University of Oxford, Oxford, UK

\subsection{6/jech-2020-SSMabstracts. 162}

Background Anthropometric and lifestyle factors may influence cancer risks through hormonal changes but the evidence to date is not conclusive. We therefore investigated associations of body size and composition, physical activity and sedentary time with serum hormone concentrations in pre- and postmenopausal women.

Methods Design: Cross-sectional.

Setting: UK Biobank, a large prospective cohort study involving about 500,000 adults aged between 40-69 years when recruited in 22 assessment centres between 2006 and 2010.

Participants: 20,758 pre-menopausal and 71,101 post-menopausal women, of whom 4,803 (23\%) and 15,469 (22\%) respectively had accelerometer data.

Exposures: body mass index (BMI), height, waist to height ratio, waist to hip ratio, body fat mass, trunk fat mass, selfreported and accelerometer-measured physical activity and selfreported sedentary time.

Outcomes: serum concentrations of total and calculated free oestradiol, total and calculated free testosterone, sex hormone binding globulin (SHBG) and insulin-like growth factor1 (IGF-1).

Statistical analysis: Multivariable linear regression analysis. Results The exposure-outcome associations are reported only if there was at least a $5 \%$ difference in hormone concentrations between the highest and lowest exposure groups.

In pre-menopausal women, higher BMI was associated with a lower concentration of total oestradiol $(15 \%$ difference in the highest vs. lowest BMI group, i.e., $35+\mathrm{kg} / \mathrm{m}^{2}$ vs $<22.5$ $\mathrm{kg} / \mathrm{m}^{2}$ ) and a higher concentration of calculated free oestradiol $(22 \%)$. The results for oestradiol were not available in postmenopausal women. In both pre- and post-menopausal women, higher BMI was associated with higher concentrations of total and calculated free testosterone (pre-menopausal 29\% and $113 \%$, post-menopausal $39 \%$ and $126 \%$, respectively) and lower concentrations of SHBG and IGF-1 (pre-menopausal $51 \%$ and $14 \%$, post-menopausal $51 \%$ and $12 \%$, respectively). Similar associations were observed with other measures of body size and composition except height.

Self-reported physical activity was associated with somewhat lower concentrations of total and calculated free testosterone (pre-menopausal 10\% (free testosterone), post-menopausal 5\% and $11 \%$ respectively in the most vs. least active quartile) and a higher concentration of SHBG (premenopausal 11\%, postmenopausal 10\%), and the opposite was true for self-reported sedentary time. The associations were slightly stronger with accelerometer-measured physical activity, but were attenuated after adjustment for BMI.

$\mathrm{P}_{\text {trend }}$ for all reported associations was $<0.0001$.
Conclusion This study confirms strong associations between adult anthropometric factors and hormone and SHBG concentrations in both pre- and post-menopausal women; this may partly explain the effects of these factors on cancer risks. The associations with physical activity and sedentary time were at most modest.

\section{P71 THE PROPENSITY TO CYCLE TOOL: A POLICY TOOL TO ESTIMATE CYCLING POTENTIAL FOR ENGLISH AND WELSH TRANSPORT PLANNERS}

${ }^{1} \mathrm{~J}$ Woodcock*, ${ }^{2} \mathrm{R}$ Aldred, ${ }^{3} \mathrm{R}$ Lovelace, ${ }^{4} \mathrm{~A}$ Goodman. ${ }^{1} \mathrm{MRC}$ Epidemiology Unit, University of Cambridge, Cambridge, UK; ${ }^{2}$ Active Travel Academy, University of Westminster, London, UK; ${ }^{3}$ Institute of Transport Studies, University of Leeds, Leeds, UK; ${ }^{4}$ Department of Population Health, London School of Hygiene and Tropical Medicine, London, UK

\subsection{6/jech-2020-SSMabstracts. 163}

Background Getting people cycling is an increasingly common objective in transport planning. A growing evidence base indicates that high quality infrastructure can boost cycling rates. Yet for measures to be effective, it is important to intervene in the right places. This creates the need for tools and methods to help answer the question 'where to build?'

The Propensity to Cycle Tool (PCT) www.pct.bike is an open source, freely available, interactive tool to help prioritise cycling in England and Wales, covering both commuting and travel to school. In addition to the tool the data are available as download to allow more detailed GIS analysis. It was created by an academic team for the Department for Transport. Methods The PCT uses origin data from the 2011 Census on main mode of travel to work and the 2011 National School Census covering all state-schools in England. We modelled propensity to cycle as a function of route distance and hilliness between home and school or work. We generated scenarios, e.g. 'Go Dutch' - in which people in England were as likely to cycle as theDutch, accounting for trip distance and hilliness. We did this based on a synthetic microsimulation population, allowing flexible scenarios and more accurate impact calculations.

We estimated changes in the level of cycling, walking, and driving, and associated impacts on physical activity and carbon emissions. For adults we estimated health economic benefits from reductions in premature mortality and sickness absence from increases in physical activity. Health outcomes were calculated using a bespoke and improved version of the UK Transport Appraisal Guidance (originally based on the WHO HEAT tool). Our improvements include using individual level rather than aggregate data, ebikes, integrating health gains from cycling with losses from less walking, and adjusting physical activity levels for route hilliness.

Results The PCT scenarios show the substantial potential for increases in cycling and the large benefits that this could realise. For example in 2011, 1.8\% of children cycled to school. Under the Go Dutch scenario, this would rise to $41.0 \%$. This would increase physical activity from school travel among pupils by $57 \%$, and reduce transport-related carbon emissions by 81 kilotonnes/year. These impacts would be substantially larger in secondary schools than primary schools (a 96\% vs. 9\% increase in physical activity, respectively).

Conclusion The PCT is currently used by over 60 local authorities in England and Wales, and is contributing to the development of local policies as part of the Cycling and Walking Investment Strategy. 